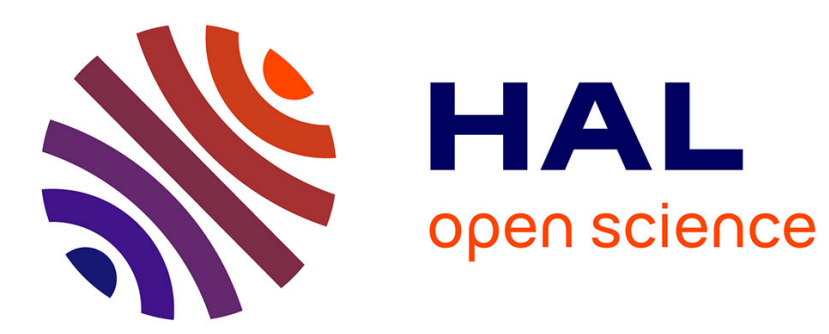

\title{
Restoration of laser-induced process kinetics from photoacoustic measurements
}

\author{
O. Puchenkov
}

\section{To cite this version:}

O. Puchenkov. Restoration of laser-induced process kinetics from photoacoustic measurements. Journal de Physique IV Proceedings, 1994, 04 (C7), pp.C7-225-C7-228. 10.1051/jp4:1994753 . jpa00253282

\section{HAL Id: jpa-00253282 https://hal.science/jpa-00253282}

Submitted on 1 Jan 1994

HAL is a multi-disciplinary open access archive for the deposit and dissemination of scientific research documents, whether they are published or not. The documents may come from teaching and research institutions in France or abroad, or from public or private research centers.
L'archive ouverte pluridisciplinaire HAL, est destinée au dépôt et à la diffusion de documents scientifiques de niveau recherche, publiés ou non, émanant des établissements d'enseignement et de recherche français ou étrangers, des laboratoires publics ou privés. 


\title{
Restoration of laser-induced process kinetics from photoacoustic measurements
}

O.V. Puchenkov(1)

N.N. Andreev Acoustics Institute, 117036 Moscow, Russia

\begin{abstract}
Reconstruction of the kinetics of relaxation processes induced in a medium by laser radiation is considered. Implementation of $\alpha$-regularization algorithm of the ill-posed problem solution in optoacoustic (OA) diagnostics of liquids is discussed. The relationship between parameters of the diagnostic experimental set-up and the frequency band where relaxation spectra can be restored is established. The restoration algorithm is applied to analysis of OA-waveforms detected in aqueous solution near $4^{\circ} \mathrm{C}$. It is shown that only broad-band detection could give necessary information for identification of particular relaxation mechanism in this case.
\end{abstract}

\section{INTRODUCTION}

Direct measurements of acoustic transients generated in a medium by pulsed laser radiation provide valuable kinetic information about relaxation processes in photoactive systems [1]. Analysis of optoacoustic $(\mathrm{OA})$ waveforms may be used also for investigation of laser-induced phase transitions and chemical reactions. Numerical algorithm one should apply to restore the dynamics of photoinduced enthalpic and volumetric changes depends substantially upon a-priori information about the nature of underlying relaxation process. In the simplest case of pure exponential decay there is explicit information about time dependence of heat release. Therefore, iterative approximation technique based on nonlinear least squares fitting of experimental data by the sum of exponentials may be applied. Time resolution of this deconvolution technique was reported to be comparable with the digitization channel width [2]. However, the approximation algorithms cannot be used to restore dynamics of complex relaxation processes with unknown kinetic scheme, for example, in the case of OA-signal generation in aqueous solutions near $4^{\circ} \mathrm{C}$ [3], or in suspension of bacterial reaction centers [4]. In these cases another algorithm that does not require preliminary information about relaxation spectra should be applied. In this report implementation of powerful $\alpha$-regularization algorithm of ill-posed inverse problem solution in pulsed OAdiagnostics of liquid samples is considered. The algorithm is applied to analysis of OA-signals generated in aqueous solution near $4^{\circ} \mathrm{C}$.

\section{OPTOACOUSTIC DIAGNOSTICS AS INVERSE PROBLEM}

General experimental procedure of OA-diagnostics includes some necessary elements. Incident laser beam is absorbed in a sample to produce excited states that relax to the initial state according to the temporal function $r(t)$. Only those relaxation processes that stimulate volume changes are considered in optoacoustics. Laser induced volume changes ( of thermal and non-thermal origin) generate sound waves that propagate in a medium to the input of pressure sensor. The sensor converts the acoustic signal to output transient voltage. Spectrum of the measured electric signal $U(\omega)$ may be connected to a relaxation 
spectrum $R(\omega)$ by the following expression:

$$
U(\omega)=\Gamma(\omega) G(\omega) I(\omega) R(\omega)=X(\omega) R(\omega)
$$

where $I(\omega)$ - spectrum of laser pulse intensity; $G(\omega)$ - medium transfer function which is responsible for the process of sound generation and propagation; $\Gamma(\omega)$ - transfer function of pressure sensor. Function $X(\omega)$ which translates relaxation spectrum to the spectrum of output electric signal describes all stages of OA-diagnostics and may be referred to as "optoacoustic filter"( OA-filter). The shape of this filter may be calculated for particular schemes of OA-conversion and properties of pressure sensors, or directly measured in experiment with a reference, non-photoactive solution.

The inverse problem of reconstruction of the relaxation spectrum $R(\omega)$ expressed mathematically by convolution equation (1) is known as the ill-posed problem. The necessary condition of stability is not valid for this kind of problems: arbitrary small uncertainties in determination of spectrum $U(\omega)$ lead to dramatic errors in restoration of the function $R(\omega)$. There are several possible ways to solve the inverse problem. In the case when the functional dependence of relaxation kinetics is known, one can use iterative approximation procedure to the find quantum yields and the relaxation time constants [2]. In more complex situations, where relaxation mechanisms are not understood yet, two-step approach to solution of the inverse problem is needed. First, explicit information about the kinetics of relaxation process should be found. Second, some reasonable mechanism of the process should be proposed, and then the parameters involved in theoretical scheme can be calculated by one of the nonlinear programming algorithms.

Very effective algorithm of the ill-posed problem solution which implies only continuity of the function $r(t)$ is a regularization method, namely Tikhonov's $\alpha$-regularization [5]. According to this method the restored relaxation function $\hat{\mathrm{r}}(\mathrm{t})$ is determined by:

$$
\hat{r}(t)=\frac{1}{2 \pi} \int_{-\infty}^{\infty} K(\omega) \frac{\hat{U}(\omega)}{\hat{X}(\omega)} e^{i \omega t} d \omega
$$

( symbol "A" is used to designate the experimentally measured spectra that differ from ideal ones due to noise present in the detecting system). Special stabilizing multiplier $K(\omega)$ included in this formula for $\alpha-$ regularization procedure is given by [5]:

$$
K(\omega)=\frac{|\hat{X}(\omega)|^{2}}{|\hat{X}(\omega)|^{2}+\alpha Q(\omega)}
$$

where $Q(\omega)$ - real non-negative even function of $\omega$, and $\alpha$ - parameter of regularization. The proper choice of $\mathrm{Q}(\omega)$ and $\alpha$ ensures stability of the restoration algorithm in the presence of noise and small deviations of restored function $\hat{R}(\omega)$ from the real one.

As any other linear algorithms $\alpha$-regularization can restore the spectrum $R(\omega)$ in a frequency range which is determined by the pass-band of the OA-filter $X(\omega)$ and signal-to-noise ratio $S / N$. Shape of the OA-filter should be measured on a standard reference solution. Nevertheless, it is important to know in advance how to estimate $\mathrm{X}(\omega)$ theoretically for a proper choice of OA-diagnostics set-up elements and for the preliminary evaluation of possible results of relaxation dynamics restoration. In OA-diagnostics of weakly absorbing solutions a cylindrical scheme of experimental set-up is widely used. The pressure detector is located aside of cylinder-like region of light absorption and its surface oriented along the laser beam axis. In this case frequency band of the OA-filter depends upon laser beam radius and the transfer function of acoustic sensor. Let us compare low-frequency and broad-band set-ups of OA-diagnostics experiment. We shall assume that low-frequency measurements are conducted with effective radius of gaussian cross-sectional intensity distribution $\mathrm{R}=2 \mathrm{~mm}$ and cut-off frequency of damped piezoceramic PZT-detector $\mathrm{f}_{u \mathrm{p}}=0.5 \mathrm{MHz}$ (piezoelement thickness $\approx 4 \mathrm{~mm}$ ). For broad-band measurements we chose beam radius $R=20 \mu \mathrm{m}$ and acoustically matched piezopolymer PVDF-film detector with $f_{u p}=30 \mathrm{MHz}$ (piezofilm thickness $\approx 30 \mu \mathrm{m}$ ). The shapes of correspondent OA-filters are shown in Fig. 1 by dashed lines. To find the frequency band in which relaxation spectra can be restored with a given confidence level one can apply $\alpha$-regularization algorithm in the case of $\delta$-functional relaxation with uniform spectrum $R(\omega)=1$. As it follows from Fig. 1 for each particular $S / N$ ratio there exist definite frequencies ( marked in Fig.1 
by arrows) that limit the range where restored spectrum is nearly coincident with the real one. This range
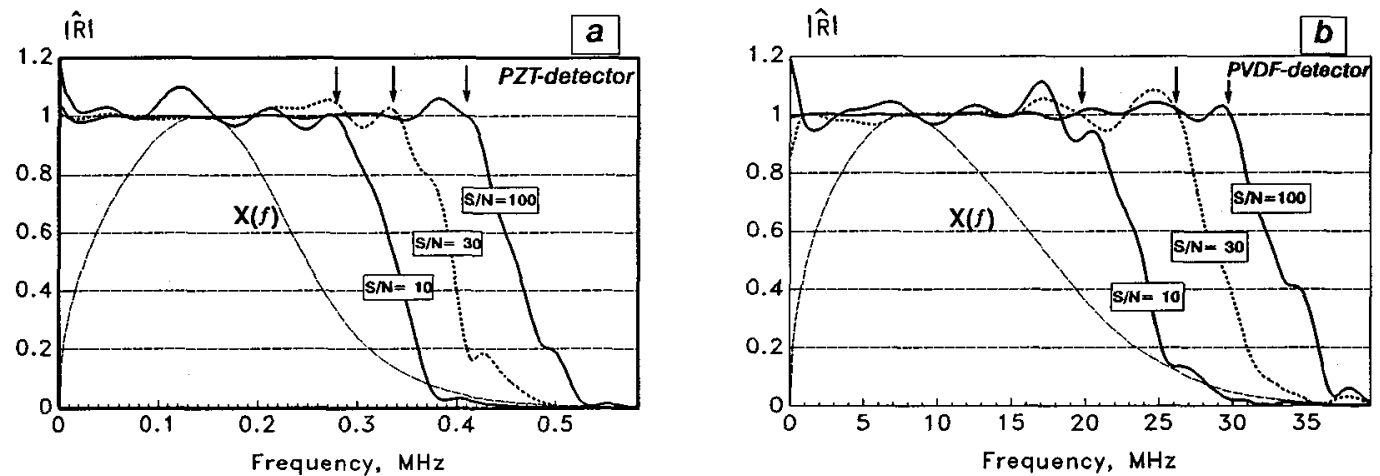

Fig.1 Spectrum of $\delta$-functional relaxation restored with different OA-filters $\mathrm{X}(f)$ ( shown by dashed line) for various $\mathrm{S} / \mathrm{N}$ ratios. (a) - low-frequency experimental set-up based on PZTdetector; (b) - broad band set-up based on PVDF-detector.

may be referred to as the frequency band of restoration. Beyond this range information about spectrum $R(\omega)$ cannot be restored by linear algorithms. The general scheme of $\alpha$-regularization algorithm implementation in OA-diagnostics of liquids with arbitrary optical absorption is considered in detail in [6]. Its performance is evaluated for several widely used experimental set-ups. In the report this algorithm is applied to the problem of OA-signal generation in water near $4^{\circ} \mathrm{C}$.

\section{ANALYSIS OF OA-SIGNALS GENERATED IN $4^{\circ} \mathrm{C}$ WATER}

Hunter and co-workers [3] reported the detection of "non-thermal acoustic signals" generated by laser radiation in aqueous solution near $4^{\circ} \mathrm{C}$ (thermal expansion coefficient of water vanishes at $4^{\circ} \mathrm{C}$ ). Later, some interpretation of the phenomenon that implies existence of new structural relaxation mechanism in $4^{\circ} \mathrm{C}$ water with relaxation time $\tau_{\mathrm{r}}=10^{-8} \mathrm{~s}$ was proposed [7]. Another possible explanation of Hunter's experiment was suggested in [8]. Observed OA-signals were attributed to the presence in sample solution of micro particles. In this case relaxation mechanism is connected with thermodiffusion through the particle/liquid interface. Acoustic signals are generated by transient changes of particle volume

$u(t) / u_{\max }$

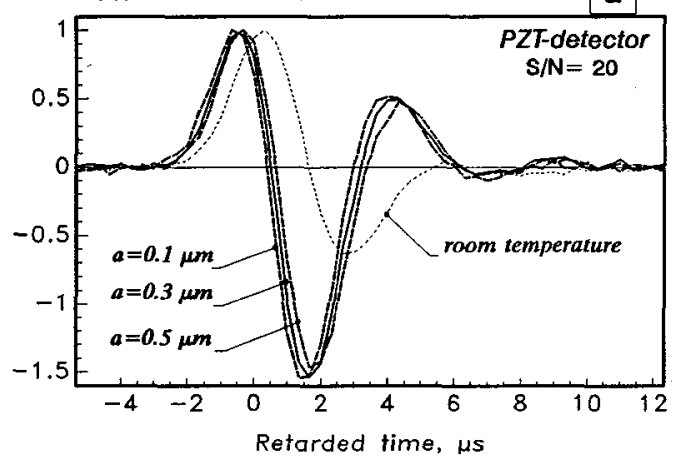

$u(t) / u_{\max }$

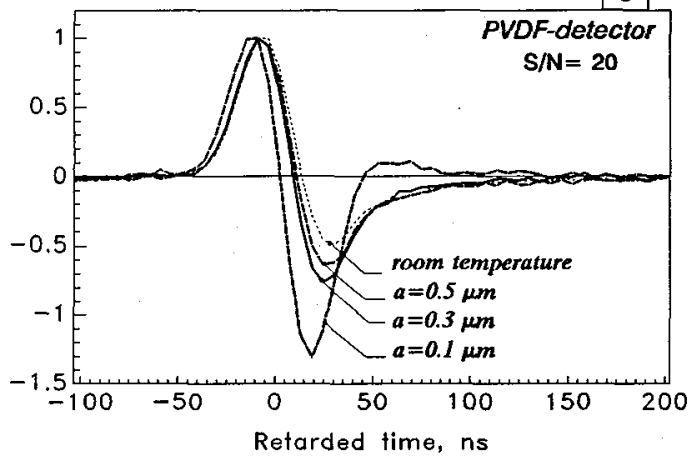

Fig.2 Normalized output transients correspondent to OA-signals generated in aqueous solution at $4^{\circ} \mathrm{C}$ for different radius $a$ of suspended particles: (a) - for low-frequency detection with PZTsensor; (b) - for broad-band detection. The signal for room temperature is shown by dashed line.

during equilibration of temperature between particle and liquid. This relaxation mechanism is characterized by a time constant which is proportional to the square of particle radius and depends upon thermophysical 
properties of particle and liquid [8]. Output voltage signals measured under the experimental conditions of [3] would be different for different OA-filters $X(\omega)$. Assume that thermophysical properties of suspended particles were equal to those of polystyrene. Then, in the case of low frequency OA-filter shown in Fig. 1a ( which corresponds to experimental set-up used by Hunter and co-workers) calculations give tripolar output signal that was actually observed in experiment [3]. Correspondent normalized waveforms are presented in Fig.2a for different particle sizes. On the other hand, for broad-band set-up (Fig. 1b) the measurements would give transients that are shown in Fig.2b. Comparison of two numerical experiments shows that low-frequency detection can not resolve particles with sizes below $0.5 \mu \mathrm{m}$ : output signals have different amplitude but nearly the same shape. For unknown particle concentration the amplitude of the signals can not be an informative parameter. Broad-band detection gives distinct difference of the signal shapes. Relaxation spectra correspondent to waveforms of Fig. 2 are displayed in Fig. 3 by dotted lines. Restored relaxation spectra for particle radius $\mathrm{a}=0.3 \mu \mathrm{m}$ are presented in Fig. 3 by solid lines.
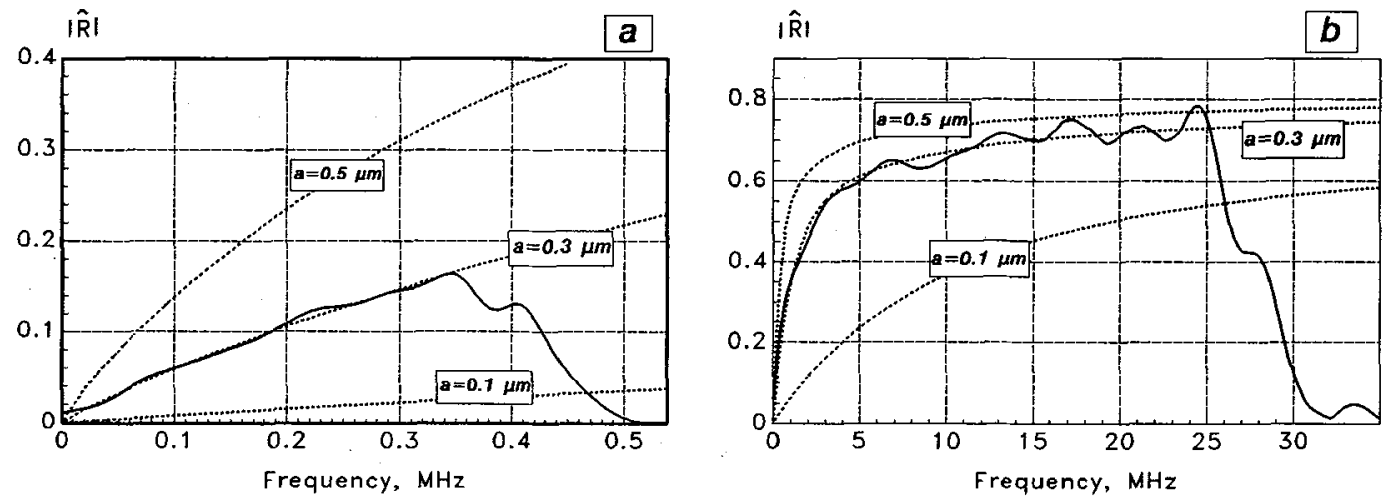

Fig.3 Relaxation spectra restored from $\mathrm{OA}$-waveform measured at $4^{\circ} \mathrm{C}$ aqueous solutions under condition of $S / N=20$ : (a) - low-frequency detection; (b) - broad-band detection.

If neither material nor size of the suspended particles are known in advance then low-frequency detection does not provide enough information to restore these parameters by nonlinear least squares fitting of the retrieved part of relaxation spectra using expressions of work [8]. At the same time, numerical analysis shows that for particle radii in the range $0.05-0.5 \mu \mathrm{m}$ broad-band detection considered here would be sufficient to find both size and thermodiffusivity coefficient of the particles.

In conclusion, low-frequency measurements carried out in [3] are hardly sufficient to identify relaxation mechanism induced by laser irradiation of $4^{\circ} \mathrm{C}$ water. More accurate broad-band measurements are necessary for restoration of relaxation spectra by the proposed $\alpha$-regularization algorithm.

\section{ACKNOWLEDGMENTS}

This work was made possible by post-doctoral fellowship of Feinberg Graduate School, The Weizmann Institute of Science. I am grateful to Prof. S.Malkin for constant support of my work, and to Dr. V.Brumfeld for critical comments on the report.

\section{REFERENCES.}

[1] Braslavsky S.E.,Heibel G.E., Chem.Rev. 92 (1992) 1381-1401.

[2] Rudzki-Small J.,Libertiny L.J.,Small E.W., Biophys.Chem. 42 (1992) 29-48.

[3] Hunter S.D.,Jones W.V., Malbrough D.J., J.Acoust.Soc.Am. 69 (1981) 1563-1567.

[4] Malkin S.,Churio M.S., Shochat S.,Braslavsky S.E., Photochem.Photobiol. (in press).

[5] Tikhonov A.N., Arsenin V.Y. Methods of ill-posed problem solution (Nauka, Moscow, 1979).

[6] Puchenkov O.V., to be published.

[7] Pierce A.D., J.Acoust.Soc.Am. 72,Suppl.1 (1982) 513-514.

[8] Zozulya O.M.,Puchenkov O.V., Sov.Phys.Acoustics 39 (1993) 46-50. 